\title{
Biodiversity of endophytic bacteria and fungi of wild grapes Vitis amurensis Rupr.
}

\author{
Olga A. Aleynova ${ }^{*}$, Nikolay N. Nityagovsky, and Konstantin V. Kiselev
}

Far Eastern Branch of the Russian Academy of Sciences, Federal Scientific Center of the East Asia Terrestrial Biodiversity FSC of Biodiversity FEB RAS, 690022, Vladivostok, Russia

\begin{abstract}
The diversity of endophytic bacteria and fungi of $V$. amurensis grape plants growing in the suburbs of Vladivostok in the summer and autumn periods of 2018-2020 was analyzed. About 600 strains of bacteria and 160 strains of fungi were inoculated from peripherally sterilized leaves and stems of $V$. amurensis. Isolated bacteria were representatives of 36 genera: Actinobacterium, Acinetobacter, Agrobacterium, Arthrobacter, Bacillus, Buttiauxella, Curtobacterium, Duganella, Erwinia, Enterobacter, Frigoribacterium, Frondihabitans, Klebsiella, Leclercia, Lelliottia, Methylobacterium, Microbacterium, Mucilaginibacter, Luteibacter, Lysinimonas, Pantoea, Paenibacillus, Parageobacillus, Pedobacter, Phyllobacterium, Plantibacter, Pseudomonas, Pullulanibacillus, Raoultella, Rhizobium, Sphingomonas, Staphylococcus, Stenotrophomonas, Streptomyces, Serratia, Xanthomonas. The largest number of strains were representatives of the genera Erwinia, Pantoae and Pseudomonas. Endophytic grape fungi were represented by 25 genera: Alternaria, Annulohypoxylon, Aureobasidium, Biscogniauxia, Cladosporium, Colletotrichum, Coniochaeta, Coprinellus, Davidiellaceae, Didymella, Discosia, Epicoccum, Fusarium, Hypoxylon, Neosetophoma, Nemania, Neurospora, Nigrospora, Paraphoma, Penicillium, Pestalotiopsis, Pestalosphaeria, Phoma, Trichoderma, Xylaria. The largest number of representatives were of the genus Didymella, Cladosporium and Colletotrichum.
\end{abstract}

\section{Introduction}

Grapes are one of the most sought-after and economically important crops in the world. In turn, this plant is quite susceptible to the effects of the external environment and diseases of various etiologies. Biotic and abiotic stresses strongly affect the growth, fruits' productivity, and quality of cultivated grape varieties, which in turn leads to economic damage to the agricultural sector [1].

At present, the causative diseases agents of the European-Asian group of grapes based on the species Vitis vinifera L. mainly growing on the territory of European states are well known. Such pathogens include Botrytis cinerea, Erysiphe necator, Plasmopara viticola, Xylella fastidiosa, Rhizobium vitis (formerly Agrobacterium vitis), Eutypa lata, and

\footnotetext{
*Corresponding author: aleynova@biosoil.ru
} 
Botryosphaeria sp., which cause diseases such as gray mould, powdery mildew (oidium), Downey Mildew (mildew), Pierce's Disease, crown gall, Esca and Petri disease or "wire stem", respectively [ 2,3$]$.

Recently, many works have appeared devoted to the study of the microbiological community's diversity inhabiting the grape plant - endophytes. They study which microorganisms inhabit different parts of the $V$. vinifera grape plant [4] using metagenome sequencing, investigate the difference between the composition of microorganisms of healthy and diseased plants [5], identify which microorganisms are capable of suppressing the growth of pathogenic microorganisms [6].

Earlier, 11 strains of endophytes obtained from the stems of $V$. vinifera cv. Corvina capable of inhibiting the growth of Botrytis cinerea were distinguished. One strain representing the genus Lysinibacillus induced inhibition of the broad growth zone of $B$. cinerea compared to other strains [7]. Recent screening has also shown that significant amounts of endophytic bacterial isolates from grapevine can inhibit the growth of Neofusicoccum parvum, Botryosphaeria dothidea, Botryosphaeria obtuse, Pochonia chlamydospora and Plasmopara viticola in vitro [7,8]. Bell et al. identified 24 endophytic strains of Enterobacter agglomerans, Rahnella aquatilis, and Pseudomonas sp., which have a strong inhibitory effect against Rhizobium vitis, the causative agent of crown gall [9].

There are also several studies showing that endophytic microorganisms of grapes contribute to plant resistance to abiotic stresses. Moreover, the endophytes' benefit does not occur directly through the induction of stress-related genes, but rather occurs by preparing the plant to activate the protective response to stress [10]. For example, Paraburkholderia phytofirmans can induce various PR genes [encoding chitinase, phenylalanine ammonia lyase (PAL), lipoxygenase (LOX), and glucanase], which protect the plant from low temperatures [11]. Endophytes release molecules that take part in the removal of reactive oxygen forms (ROF), thus helping the grapes cope with various abiotic stresses such as cold, drought or soil salinity. It has been shown that the grape endophyte Bacillus licheniformis stimulates the production of secondary metabolites such as monoterpenes exhibiting antioxidant activity, and sesquiterpenes exhibiting antibacterial properties [12]. B. licheniformis produces carotenoids that can act as antioxidants [13]. It is noteworthy that these compounds are precursors of abcisic acid (ABA) in plants, which serves as the basis for plant drought resistance [14]. Endophytic fungi Septglomus deserticola, Funneliformis mosseae, Rhizoglomus intraradices, Rhizoglomus clarum, and Glomus aggregatum can also modulate ABA metabolism in inoculated grape plants, which gives them an advantage over uninfected plants under drought conditions [15].

It has also been shown that grape endophytes have a beneficial effect on the quality of grapes. For example, inoculation of grapes with the endophytes Acinetobacter lwoffii, Bacillus subtilis and Pseudomonas fluorescens active against Botrytis cinerea lead to the accumulation of stilbenes, especially trans-resveratrol (3,5,4'-trihydroxystilbene) and its oligomer, trans- $\varepsilon$-viniferine, which in turn accumulate in grapes [16].

At present, little is known about natural pathogens and especially endophytic microorganisms of the Asian group of grapes, the main representative of which is the wild Amur grape Vitis amurensis. It is known that this species tolerates low temperatures well and has a relatively high resistance to pathogens $[17,18]$. $V$. amurensis is also used as rootstocks to create grape varieties that are resistant to biotic and abiotic stress. Therefore, the main purpose of this study was to study the microbiological community of wild grapes $V$. amurensis for further creation of promising biological products for the cultivation of agricultural grape crops. 


\section{Materials and methods}

\subsection{Isolation of $V$. amurensis endophytic bacteria and fungi strains}

Stems and leaves of the wild-growing $V$. amurensis grape (young 7-8 $\mathrm{cm}$ long stems with three healthy leaves) were collected from an unprotected natural zone near the city of Vladivostok (south of Primorsky Krai of the Far East of Russia, longitude 43.19276 latitude 131.92278) in June - September 2018-2020. Each plant sample was delivered to the laboratory within 30 minutes; $1.5 \mathrm{~g}$ of leaf and stem tissue, respectively, were washed under running water with soap, then they were washed in $75 \%$ alcohol under sterile conditions for $2 \mathrm{~min}$, and then in 10\% hydrogen peroxide solution for $1 \mathrm{~min}$ and washed with sterile water 5 times. To test the effectiveness of the chosen surface sterilization method, $100 \mu \mathrm{l}$ of the last wash water was taken and pulverized on Petri dishes with potato dextrose agar (PDA) (Neogene, UK) and R2A agar media [19] to check for the absence of microorganism colonies' growth. No growth of microorganisms was detected after a three-day incubation of the last wash water on dishes with nutrient media, which indicates a high-quality surface sterilization of grape tissues. The surface sterilized tissue of leaves and stems of wild grapes was crushed in a sterile mortar to a homogeneous state, the juice was squeezed out, and $100 \mu \mathrm{L}$ of the resulting juice was applied to Petri dishes with PDA and R2A media. After three days, cultured colonies were harvested and carefully transferred to a new sterile dish for another cultivation.

\subsection{Identification of $\boldsymbol{V}$. amurensis endophytic bacteria and fungi strains}

DNA isolation of individual colonies of bacteria and fungi was carried out by the method of hexadecyltrimethylammonium bromide (CTAB) with modifications [20]. The 16S rRNA gene sequences were amplified with universal primers 926F, 5'AAC TCA AAG GAA TTG ACG G and 1522R, 5'AAG GAG GTG ATC CAR CCG CA. Universal primers 5'AGG AGA AGT CGT AAC AAG G and 5'TCC TCC GCT TAT TGA TAT GC were used to amplify the products of the ITS1 intergenic spacer [21]. PCR products were sequenced using an ABI 3130 genetic analyzer "Applied Biosystems" (USA) in accordance with the manufacturer's instructions. The sequence was analyzed using the Basic Local Alignment Search Tool (BLAST). Multiple sequence alignments were performed using the ClustalX program [22]. Sequence identity $\geq 99 \%$ is considered a sufficient cut-off value for taxonomic identification.

\section{Results and discussion}

The diversity of endophytic bacteria and fungi of two visibly healthy wild and one visibly diseased $V$. amurensis grape plants growing in the suburbs of Vladivostok in the summer and autumn periods of 2018-2020 was analyzed. Juice was isolated from peripherally sterilized leaves, stems, berries, and seeds of $V$. amurensis under sterile conditions, which was subsequently pulverized on dishes with universal nutrient media R2A (Reasoner and Geldreich, 1985) and PDA (Neogene, UK). In total, about 25 thousand colony-forming units (CFU) of bacteria were sown, from which 600 strains of bacteria were isolated. $210 \mathrm{CFU}$ of fungi were also sown, of which 173 strains of fungi were isolated. Next, DNA was isolated from the obtained strains of bacteria and fungi, PCR was placed on the $16 \mathrm{~S}$ rRNA areas for bacteria and the ITS1 intergenic spacer area for fungi, the resulting PCR products were sequenced, and a comparative analysis was performed with the previously known $16 S$ and ITS1 sequences in GenBank. 
During the analysis of the sequences, it was demonstrated that the isolated bacterial strains of wild grapes $V$. amurensis were from 36 genera: Actinobacterium, Acinetobacter, Agrobacterium, Arthrobacter, Bacillus, Buttiauxella, Curtobacterium, Duganella, Erwinia, Enterobacter, Frigoribacterium, Frondihabitans, Klebsiella, Leclercia, Lelliottia, Methylobacterium, Microbacterium, Mucilaginibacter, Luteibacter, Lysinimonas, Pantoea, Paenibacillus, Parageobacillus, Pedobacter, Phyllobacterium, Plantibacter, Pseudomonas, Pullulanibacillus, Raoultella, Rhizobium, Sphingomonas, Staphylococcus, Stenotrophomonas, Streptomyces, Serratia, Xanthomonas. The largest number of strains were representatives of the genera Buttiauxella (2.5\%), Curtobacterium (5.5\%), Erwinia (23\%), Frigoribacterium (3.9\%), Pantoae (19\%), Pseudomonas (15\%), Sphingomonas (3.9\%), Phyllobacterium (2.5\%) and Xanthomonas (2.5\%) of the total number of analyzed bacterial strains (Fig. 1). It should be noted that the quantitative and qualitative composition of the $V$. amurensis grape bacterial community varied significantly depending on the season of the year, and also differed in a visibly diseased plant from healthy $V$. amurensis grape plants. It was noted that the main part of the bacterial community of a visibly diseased plant was represented by bacteria of genera Agrobacterium (9.6\%), Bacillus (3\%), Erwinia (3.2\%), Pantoae (45\%), Pseudomonas (14\%), and Rhizobium (27\%).

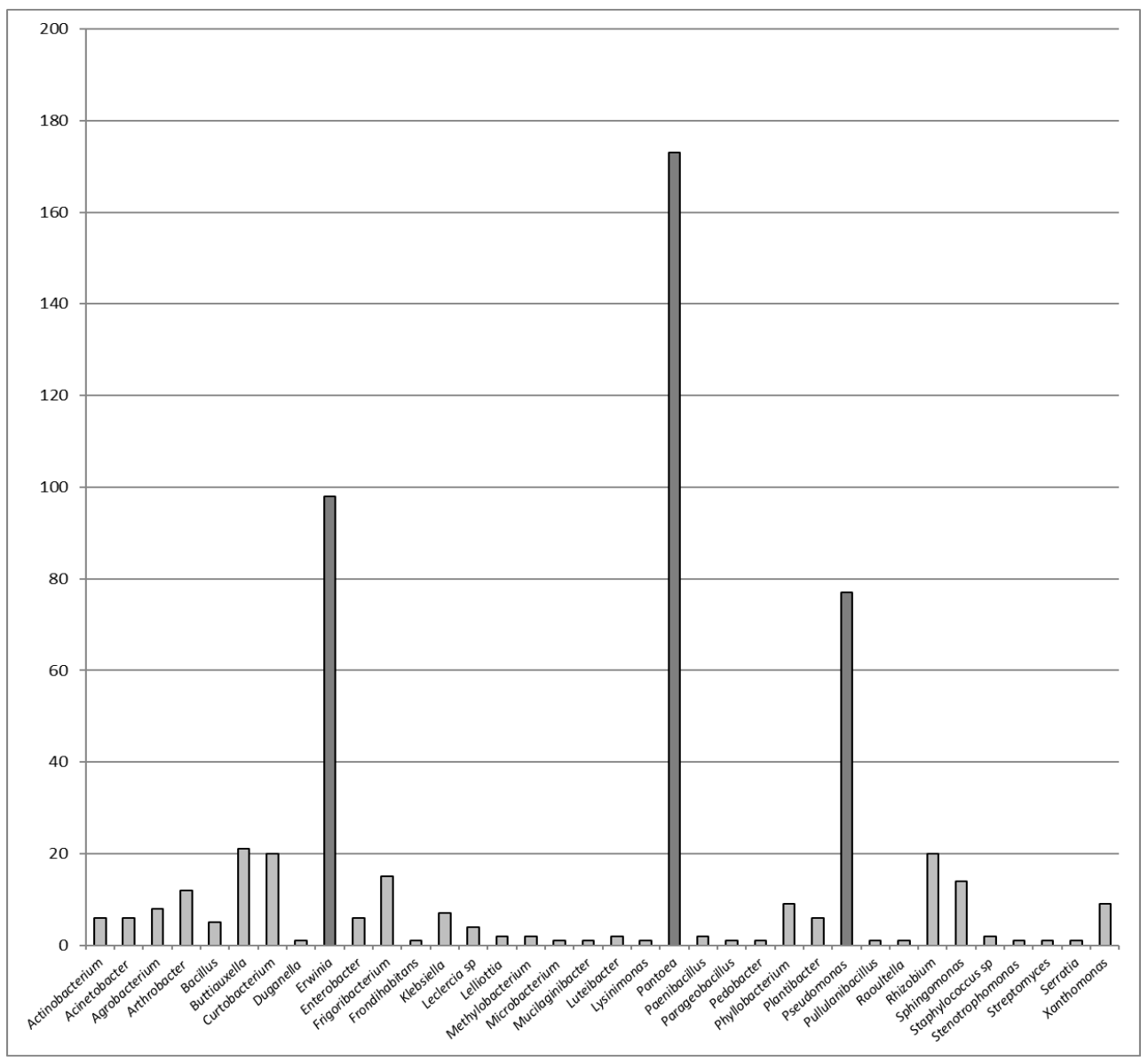

Fig. 1. Percentage ratio of endophytic bacteria genera from the total number of analyzed strains of endophytic bacteria in a healthy $V$. amurensis grape plant. The most common endophytic bacteria are marked in dark gray. 
Earlier, colleagues showed that 26 isolates were able to inhibit the growth of Botrytis cinerea on grape leaves, and 9 strains showed an antifungal effect in vitro; among them were two strains of Pantoea sp. and Pseudomonas fluorescens [23]. In addition, 25 endophyte strains isolated from domesticated and wild grape plants were very active against $B$. cinerea in vitro, especially strains belonging to genera Bacillus and Pantoea [8]. It was shown that the ability of Bacillus phytofirmans to inhibit infection of grape plants with $B$. cinerea was associated with the induction of temporary alkalization of the extracellular space, the production of salicylic acid, and the expression of transcripts associated with plant defense [24]. All of the above bacteria were also found in the course of this study in the wild-growing $V$. amurensis grape.

As a result of processing the obtained ITS1 sequences of isolated endophytic fungi strains $V$. amurensis, they were divided in 25 genera: Alternaria, Annulohypoxylon, Aureobasidium, Biscogniauxia, Cladosporium, Colletotrichum, Coniochaeta, Coprinellus, Davidiellaceae, Didymella, Discosia, Epicoccum, Fusarium, Hypoxylon, Neosetophoma, Nemania, Neurospora, Nigrospora, Paraphoma, Penicillium, Pestalotiopsis, Pestalosphaeria, Phoma, Trichoderma, Xylaria. The largest number of representatives belonged to the genus Didymella (20.8\%), Cladosporium (16.8\%) and Colletotrichum (6.9\%) (Fig. 2).

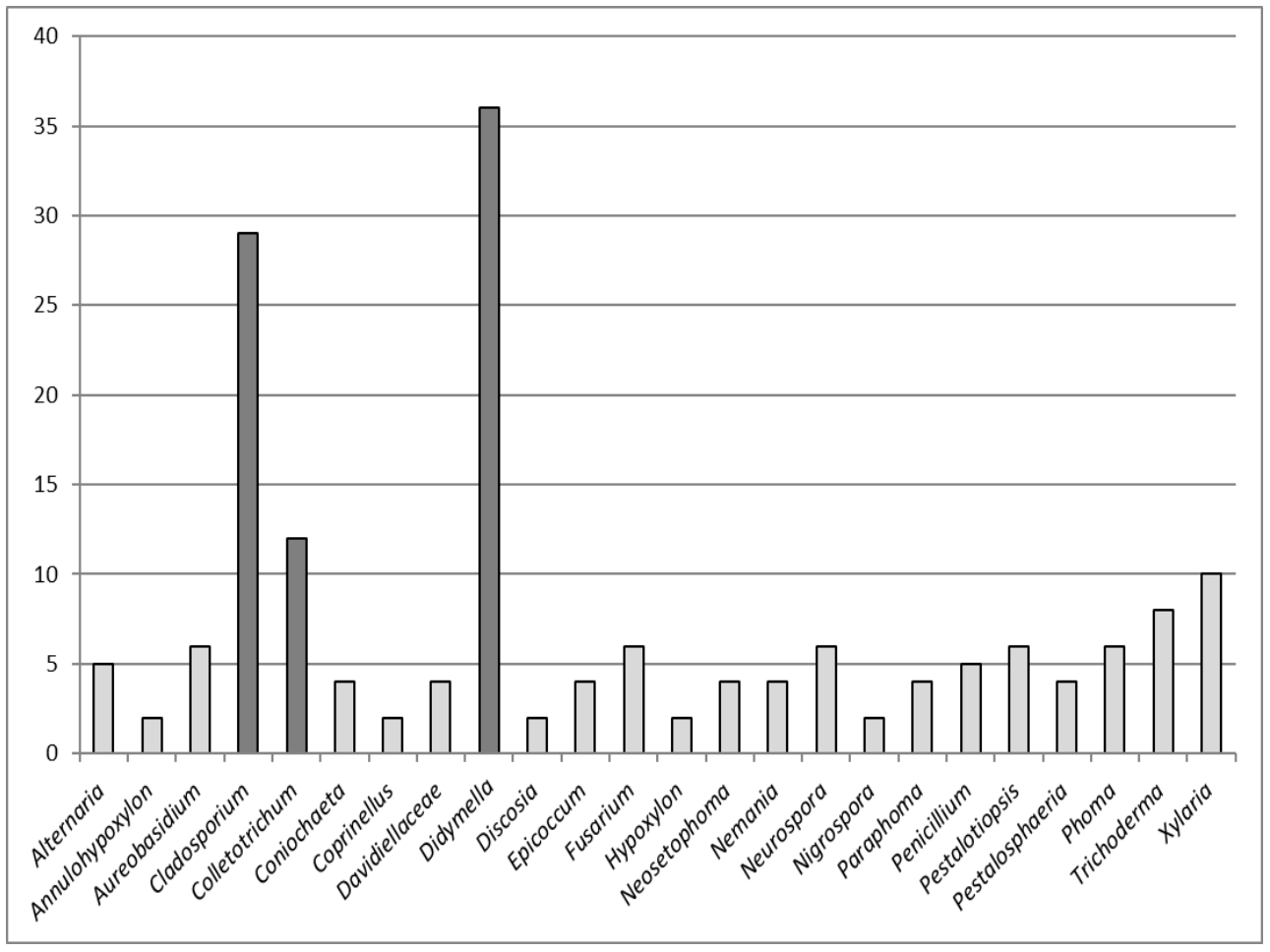

Fig. 2. Percentage ratio of endophytic bacteria genera from the total number of analyzed strains of endophytic bacteria in a healthy $V$. amurensis grape plant. The most common endophytic fungi are marked in dark gray.

It is known that most species of the genus Alternaria are facultative specific parasites that occur on all plant organs causing spotting. It was previously shown that A. alternata causes fruit rot of grapes in Pakistan [25]. Some representatives of the genus Cladosporium are capable of causing cladosporium rot of grapes ( $V$. vinifera), which is a common disease in Chile, especially in Cabernet Sauvignon and other red wine grape varieties [26]. A representative of the genus Fusarium, namely F. proliferatum, is a pathogen that causes fruit 
rot on vines in Pakistan, China and Russia [18, 25, 27]. In addition to the known facts about the pathogenicity of the genera representatives identified by the authors, representatives of the genera were found which are interesting from the point of view of biotechnology. Thus, Chinese and Indian scientists published works showing that endophytic fungi of the genera Alternaria and Xylaria isolated from $V$. vinifera grapes contain resveratrol and its derivatives, which are valuable biologically active substances for human health [28, 29].

Thus, bacterial and fungal endophytes inhabiting the $V$. amurensis grape plants are interesting for further study of their properties, which in the future may lead to the creation of biotechnological preparations used in various spheres of human life.

\section{Conclusion}

Recently, researchers have begun to pay quite a lot of attention to the study of endophytes of various plants. The practical interest in endophytic microorganisms is associated, first, with the assessment of the possibility of their use in the production of biological products to increase the productivity of cultivated plants, protect them from phytopathogenic microflora, and improve the quality of the crop.

In this work, the generic composition of endophytic bacteria and fungi of the wild Amur grape $V$. amurensis has been studied for the first time. It was found that the bacterial isolates belong to 36 genera: Actinobacterium, Acinetobacter, Agrobacterium, Arthrobacter, Bacillus, Buttiauxella, Curtobacterium, Duganella, Erwinia, Enterobacter, Frigoribacterium, Frondihabitans, Klebsiella, Leclercia, Lelliottia, Methylobacterium, Microbacterium, Mucilaginibacter, Luteibacter, Lysinimonas, Pantoea, Paenibacillus, Parageobacillus, Pedobacter, Phyllobacterium, Plantibacter, Pseudomonas, Pullulanibacillus, Raoultella, Rhizobium, Sphingomonas, Staphylococcus, Stenotrophomonas, Streptomyces, Serratia, Xanthomonas. The largest number of strains were representatives of the genera Erwinia, Pantoae and Pseudomonas. Endophytic grape fungi were represented by 25 genera: Alternaria, Annulohypoxylon, Aureobasidium, Biscogniauxia, Cladosporium, Colletotrichum, Coniochaeta, Coprinellus, Davidiellaceae, Didymella, Discosia, Epicoccum, Fusarium, Hypoxylon, Neosetophoma, Nemania, Neurospora, Nigrospora, Paraphoma, Penicillium, Pestalotiopsis, Pestalosphaeria, Phoma, Trichoderma, Xylaria. The largest number of representatives were of the genus Didymella, Cladosporium and Colletotrichum.

The obtained data is important and will allow to constrain and timely fight against pathogens in grape nurseries of the Far East; also, the results on the species composition of endophytes of Amur grapes can be used to create environmentally friendly plant protection products based on endophytic microorganisms against grape diseases of various etiologies. The development and active application of such approaches will undoubtedly contribute to the transition to highly productive and environmentally friendly agriculture.

\section{Acknowledgments}

This work was supported by the Russian Science Foundation, grant no. 20-74-00002.

\section{References}

1. R. Mzid, C. Marchive, D. Blancard, L. Deluc, F. Barrieu, M.-F. Corio-Costet, N. Drira, M. Pancher, M. Ceol, P. Corneo, C. Longa, S. Yousaf, I. Pertot, A. Campisano, Appl. Environ. Microbiol. 78(12), 4308-4317 (2012)

2. G. Armijo, R. Schlechter, M. Agurto, D. Muñoz, C.P. Nuñez, Front Plant Sci. 7, 382 (2016) 
3. R. Smart, Wine \& Viticul J. 30(5), 44 (2015)

4. S. Compant, V. Mondello, G. Conigliaro, D. Angeli, S. Micheli, M. Maurhofer, Biocontrol of Major Grapevine Diseases: Leading Research. 182-190 (2016)

5. D. Bulgari, P. Casati, F. Quaglino, P. Bianco, BMC Microbiol. 21(14), 198 (2014)

6. A. Shcherbakov, S. Mulina, P. Rots, E. Shcherbakova, V. Chebotar, Agronomy Research. 14(5), 1702-1712 (2016)

7. M. Andreolli, S. Lampis, G. Zapparoli, E. Angelini, G. Vallini, Microbiol. Res. DOI: 10.1016/j.micres.2015.11.009 (2016)

8. A. Campisano, M. Pancher, G. Puopolo, A. Puddu, S. Lopez-Fernandez, B. Biagini et al. Am. J. Enol. Vitic. 66, 12-21, doi:10.5344/ajev.2014.14046 (2015)

9. C. R. Bell, G. A. Dickie, W. L. G. Harvey, J. W. Y. F. Chan, Can. J. Microbiol. 41, 4653 (1995)

10. Q. Esmaeel, M. Pupin, N. P. Kieu, G. Chataigné, M. Béchet, J. Deravel, et al. MicrobiologyOpen 5 (3): 512-526 (2016)

11. A. Theocharis, S. Bordiec, O. Fernandez, S. Paquis, S. Dhondt-Cordelier, F. Baillieul, et al. Mol. Plant Microbe Interact. 25, 241-249 (2012)

12. M. V. Salomon, R. Bottini, G. A. de Souza Filho, A. C. Cohen, D. Moreno, M. Gil, et al. Physiol. Plant. 151 (4), 359-374 (2014)

13. A. C. Cohen, E. Dichiara, V. Jofré, A. Antoniolli, R. Bottini, P. Piccoli, Int. J. Food Sci. Tech. 53 (12), 2697-2705 (2018)

14. M. V. Salomon, R. Purpora, R. Bottini, P. Piccoli, Plant Physiol. Biochem. 106, 295304 (2016)

15. N. Torres, N. Goicoechea, A. M. Zamarreño, M. Carmen Antolín, Plant Sci. 274, 383393 (2018)

16. B. Verhagen, P. Trotel-Aziz, P. Jeandet, F. Baillieul, A. Aziz, Phytopathol. 101, 768777 (2011)

17. Q. Chen, L. Diao, H. Song, X. Zhu, Phytomedicine, 49, 111-122 (2018)

18. Yi. Wang, H. Xin, P. Fan, J. Zhang, Y. Liu, Y. Dong, Z. Wang, Y. Yang, Q. Zhang, R. Ming, G.Y. Zhong, Sh. Li, Zh. Liang, The Plant J. 105, 1495-1506 (2021)

19. D. J. Reasoner, E. E. Geldreich, Appl. Environ. Microbiol. 49(1), 1-7 (1985)

20. K.V. Kiselev, A.P. Tyunin, Y.A. Karetin, Plant Cell Rep. 34, 311-320 (2015)

21. E. Deyett, P. E. Rolshausen, FEMS Microbiology Ecology, 96, 053 (2020)

22. S.F. Altschul, W. Gish, W. Miller, E.W. Myers, D.J. Lipman, J. Molecul. Biology, 3, 403-410 (1990)

23. P. Trotel-Aziz, M. Couderchet, S. Biagianti, A. Aziz, Environ. Exp. Bot. 64, 21-32 (2008)

24. E. Ait-Barka, A. Belarbi, C. Hachet, J. Nowak, J. C. Audran, FEMS Microbiol. Lett. 186, 91-95 (2000)

25. S. Ghuffar, G. Irshad, M. Shahid, F. Naz, A. Riaz, M. A. Khan, N. Mehmood, A. Sattar, H. M. Asadullah, M. L. Gleason, Plant diseases, 102(8) (2018)

26. E. X. Briceño, B. A. Latorre, Plant Disease 92(12), 1635-1642 (2008)

27. E. G. Yurchenko, N. V. Savchuk, E. V. Porotikova, S. V. Vinogradova, Plant Disease, 104(3), (2019)

28. Ya. Lu, Ch. Ye, J. Che, Xi. Xu, D. Shao, Ch. J. Y. Liu, J. Shi, Microb Cell Fact. 18, 13 (2019) 
29. V. Dwibedi, S. Kalia, S. Saxena, Mol. Biol. Res. 46 (4), 4123-4137 (2019) 